\title{
New Science at the Meso Frontier: Dense Nanostructure Architectures for Electrical Energy Storage
}

\author{
Gary W. Rubloff ${ }^{1,2}$ and Sang Bok Lee ${ }^{3}$ \\ ${ }^{1}$ Department of Materials Science and Engineering, University of Maryland, College Park, MD 20742 \\ ${ }^{2}$ Institute for Systems Research, University of Maryland, College Park, MD 20742 \\ ${ }^{3}$ Department of Chemistry and Biochemistry, University of Maryland, College Park, MD 20742
}

Corresponding author:

Gary Rubloff, rubloff@umd.edu, 301-405-3011

Institute for Systems Research, 2145 AV Williams Bldg, University of Maryland, College Park, MD 207423285

\section{Abstract}

We examine the scientific challenges and opportunities presented at the mesoscale in the context of employing nanostructures for electrical energy storage. In order to capitalize on the power-energy and charge/discharge cycling stability that nanostructures offer, massive assemblies of nanostructures in networks must be organized into dense mesoscale architectures. With a fairly wide variety of architectures already demonstrated and more expected, the essential questions are whether regular or random 3-D arrangements are favorable, which embodiments should show best performance, and at what dimensional scaling? Dense packing raises challenging new questions about ion available and transport in highly confined electrolyte nanoenvironments, as well as designs to balance ion transport in electrolyte and electron transport in electrodes over distances long compared to nanostructure characteristic dimensions. Architectures and dimensional scaling present important issues of defects, statistical outliers, and their dynamic evolution, which in turn control degradation and failure phenomena. These considerations promise a rich set of mesoscale scientific challenges crucial to exploiting storage nanostructures in mesoscale architectures for energy storage.

Keywords: energy storage; mesoscale; nanostructures; ionics; electrodics capacity degradation; defects

\section{Motivation - from nano to meso}

The march of nanoscale science and technology ("nano" for short) over the past $\sim 2$ decades, underwritten by national and global initiatives and support, has been a central focus for research and development. It has driven, and has been driven by, advances in our ability to synthesize, characterize, model, achieving new levels of performance in metrics relating to nanomaterials, nanostructures, and their applications. The intensity and rate of progress in nano shows little if any sign of abating, nor should it given that the nanoscale is where much of nature's secrets lie, and where our ability likely resides to reconfigure and exploit these secrets for societal benefit.

Nevertheless, it is natural to ask:

(Q1) Are there new scientific challenges beyond or in front of the nano domain?

(Q2) Are there applications of nano yet to be exploited?

(Q3) What is the link to these challenges and benefits?

Rubloff, Lee: New Science at the Meso Frontier: Dense Nanostructure Architectures for Electrical Energy Storage 
In this opinion article, we argue in the context of energy storage that the answer to both Q1 and Q2 is a vehement yes, and that the link in Q3 is mesoscale science and technology. In doing so, we adopt the definition of "mesoscale" as employed in the "MESO Report", i.e., the 2012 DOE Office of Science report "From Quanta to the Continuum: Opportunities for Mesoscale Science"[1], where "meso" refers to properties and behavior across multiple length, time, and functionality regimes.

For our purposes, by "meso" we focus on an ensemble of nanoscale constructs, realizing a system of smaller things which together exhibit (a) new kinds of behavior, either good or bad, not simply the sum of individual components, and representing new science (Q1), and (b) viable functionality as a nanostructure-based system with value at the micro or macro scale, leading to beneficial applications of nano (Q2).

Here we explore these ideas through the example of nanostructures for energy applications, particularly as applied to electrical/electrochemical energy storage (EES). Recent EES research has emphasized the role of nanostructures and nanomaterials to enhance performance, focusing both on the design of component materials

[2-7] [8] [9] [10-13] and nanostructures[14-26], and on the architectures[25,27]

[28-38] in which they are assembled at the mesoscale, all of which have a strong influence on EES performance metrics. The spectrum of nanostructures and architectures under exploration is broad, ranging from attempts to spatially downscale the size of composite particulate electrodes from micro to nano scale (typically involving binder and electrically conductive additives) to more carefully controlled shapes and sizes of electrode nanostructures with specific properties beneficial to charge transport and stability. In our DOE Energy Frontier Research Center "Nanostructures for Electrical Energy Storage (NEES)", we have adopted the mission of creating and investigating precisely controlled model nanostructures as individual, isolated components and as dense nanostructure arrays in order to identify important design features and the fundamental mechanisms which make them critical.[25]

The ability to create and compare model nanostructures in isolation vs. within dense arrays provides profound opportunity for EES research at the mesoscale. For example, new science will likely emerge when controlled nanostructured electrodes are packed closely together, altering ion and electron transport and inviting interaction between them, i.e., "ionics and electrodics". New ion transport phenomena occur at dimensions of the electrical double layer, while electron transport is altered in very thin conductive layers [39]. As another example, control in assembling precision nanostructures into regular/ordered vs. pseudo-random 3-D mesoscale architectures may provide insights and opportunities for architectural designs that benefit the performance of these "mesoscale systems" and/or minimize degradation and failure mechanisms. These kinds of considerations suggest that the value from research in EES nanostructures may be substantially realized at mesoscale levels of nanostructure aggregation, and only if new science at the mesoscale is directly addressed.

At this point, caveats concerning semantics are in order. First, "mesoscale" carries different meanings within different research communities. E.g., IUPAC, the International Union of Pure and Applied Chemistry, defines "mesoscale" and "mesopore" [40] on the basis of intermediate size or length scale, and surely the latter is familiar to a cadre of nano researchers. The significance of science at the mesoscale and applications derived from it loom far larger when "mesoscale" implies architectural design and functional behavior as aggregated systems, as well as length (and time) scales.

While this broader notion of "meso" may be new, it is likely to become accepted as the focus on the architectural design of nanostructure assemblies increases. It is comforting to recall this history of "nano" as an identifiable research topic. When it became common parlance, many correctly regarded it as just a new name for what they'd been doing already, and after a few years of overuse (i.e., hype) the term became useful to describe and stimulate significant research. In a similar fashion, identifying and pursuing meso research is likely to reveal new science challenges (Q1) and pathways to apply advances in nano (Q2).

\section{Nanostructures for storage}

Given a materials set, nanostructures offer two profound advantages - higher power and more robust charge/discharge cycling. First, by distributing active storage materials for anode and cathode

Rubloff, Lee: New Science at the Meso Frontier: Dense Nanostructure Architectures for Electrical Energy Storage 
in thin layers over larger surface areas, more energy is available at a given power, or a given energy can be delivered at higher power. This is depicted in Fig. 1, where the same mass of ion storage material on planar electrodes in (a) can be redistributed as thinner layers in nanowire structured electrodes in (b). Ion transport into electrode materials is slow, requiring times $\tau$ that scale roughly as $\tau=x^{2} / 2 D$, where $x$ is the layer thickness and $D$ the ion diffusivity, so the nanostructure achieves faster ion diffusion, i.e. higher power for the same mass of storage material. Achieving this requires fast electron transport as well, leading to a nanostructured current collector for fast electron transport to all regions of the active storage material. The example of regular, parallel nanoelectrodes depicted in Fig. 1b is only one of several types of architectures that offer enhanced power and cycle stability; others include nanoparticulate electrodes formed as planar structures like Fig. 1a, as well as a variety of 3-D random[41] and ordered [34,42-44] nanoporous scaffolds coated conformally by the functional layers.

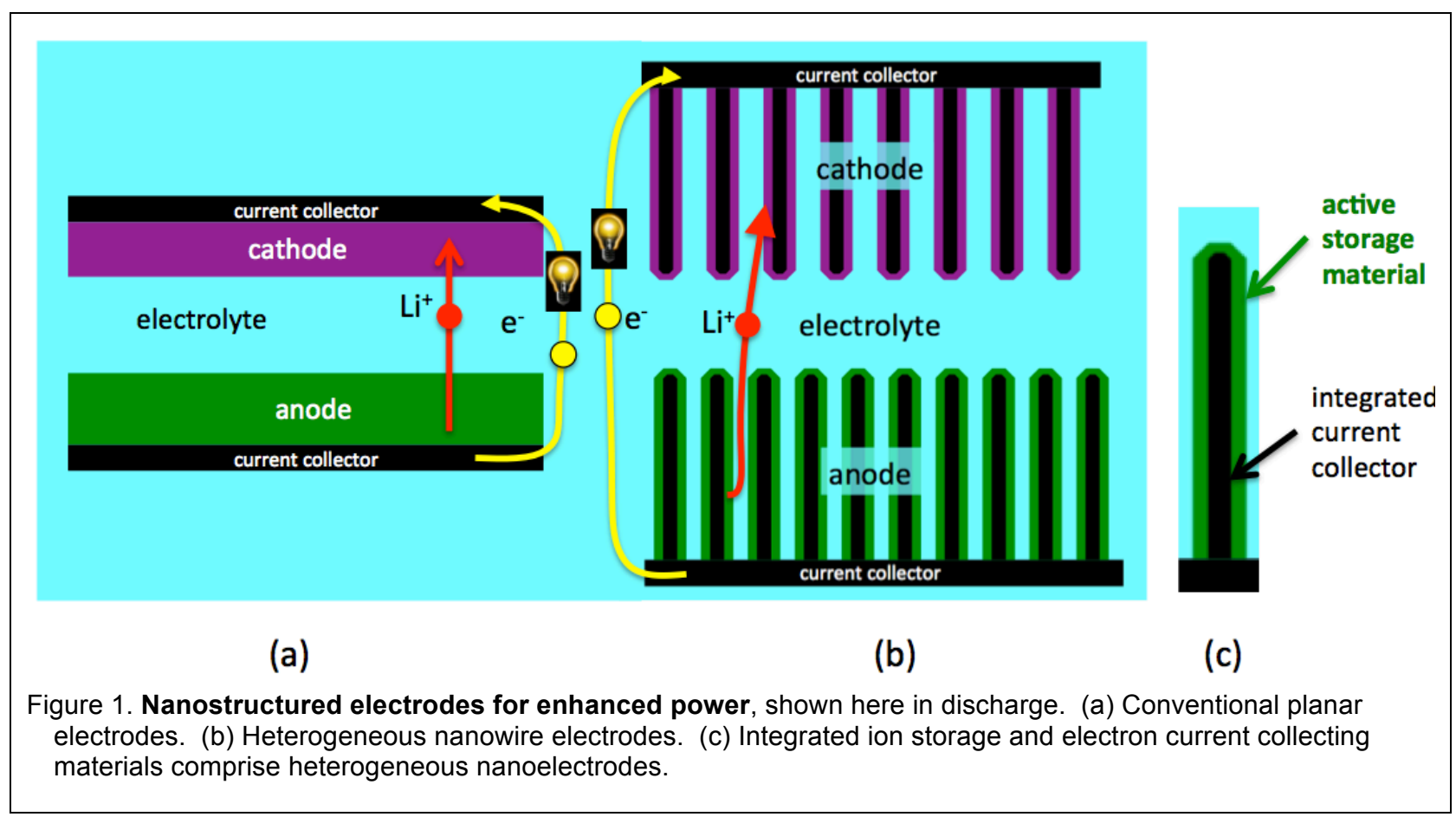

The second benefit of nanostructuring is that, properly designed, the nanowire structures are mechanically more flexible and thus can better accommodate volume change and stresses associated with insertion/deinsertion of ions during charge/discharge cycling. The importance of this feature is underscored by the fact that batteries typically lose capacity with cycling, in part because of such mechanical stresses.

Thus, nanoscale synthesis methods enable the nanostructures to obey important design guidelines thin active ion storage layers for ion transport and storage, integrated current collecting components fore fast electron transport, and strong chemical/mechanical bonds at the interface between these two materials for charge/discharge cycling stability. Effective mesoscale assemblies of such nanostructures should therefore retain these local (short-range) characteristics in longer-range 3-D architectures.

A potential drawback for high power nanostructure designs is that larger amounts of surface area are exposed to electrolyte, providing more opportunity for defects of some kind that cause degradation or failure. Since it is unlikely that new materials can be found with major improvements $(\sim 100 X)$ in ion transport rates, such nanostructuring seems the only alternative. Conformal thin film coatings onto nanostructured scaffolds, achieved by wet or dry deposition processes, are promising in this regard, and the ultrahigh aspect ratios ( $A R=$ height/width of features) employed for years in semiconductor electronics provide strong evidence that thin film approaches can manage defect control in high AR structures. 


\section{Ionics and electrodics}

A direct consequence of assembling nanostructures into dense mesoscale architectures is that ion and electron transport may be altered in fundamental ways, as illustrated in Fig. 2. In geometrically restricted electrolyte environments between closely spaced nanoelectrodes of very high aspect ratio (height/width), there may be insufficient ion population to utilize the storage material in the electrode, and ion diffusion from bulk electrolyte above the nanoelectrodes may be too slow to contribute at high power.[45] lon transport may be further modified by surface charge on the electrodes and by the narrow dimensions, particularly as they approach the length scale of the electrical double layer intrinsic to electrode/electrolyte interfaces. [46-49]

For nanostructure-based EES, length scales play a key role in achieving simultaneous high power and energy. In the simple example in Fig. 3 , this generates a design challenge to specify thicknesses of current collector, ion storage layers, and interelectrode spacing (filled by electrolyte). In a simple picture, the relative thicknesses of current collector and storage layer should be determined by their material properties, their electron and ion transport kinetics, and the design point for power-vs-energy densities. Then electrode spacing and electrolyte ion concentration may be chosen consistent with this design point. However, for long nanostructures, electron transport may be modified by grain structure and interface scattering which vary significantly with thickness in the nanoscale regime.[39] At high powers this may create voltage drops along the nanostructure, leading to inhomogeneous surface charge and ion insertion/deinsertion flux also along the nanostructure. With surface charge present, and possibly complicated by gradients in the surface charge, the electrolyte may experience electrokinetic flow effects, as well as rectification phenomena as interelectrode spacing approaches electrical double-layer overlap conditions.

These scenarios are admittedly speculative, and much more experimental and theoretical study is required to assess the magnitude of these or other ionics and electrodics effects. Nevertheless, if significant changes in ion and electron transport are indeed induced by dense spacing between nanostructures, they will constitute a profound consequence of nanostructure aggregation at the mesoscale, and their understanding and control will be a prerequisite for realizing the benefits of nanostructure-based EES.

\section{Dense nanostructure assemblies as mesoscale architectures}

For nanostructured EES to be a promising technology, the nanostructures must be packed densely so that gravimetric, volumetric, and areal energy density and power are high. The example of parallel nanoelectrodes in Fig. 1b then resembles a nanostructure "forest", while other configurations of dense nanostructures might suggest other metaphors, e.g. nanostructure "sponges" for random-porosity configurations. In any case, the requirement of dense nanostructure assemblies drives the mesoscale science challenges in electrical energy storage, presenting significant scientific and engineering questions of architectural design, ion and electron transport, statistical distributions, defects, degradation, and safety. Engineering of these architectures clearly raises questions of design optimization, from a suitable volume fraction for electrolyte, even in regular/ordered arrangements, to the performance consequences of convoluted pathways as found in random 3-D configuration, where pathways for both electrons and ions can be quite tortuous. The way in which these issues play out 
presents important science challenges, ranging from how local variations in random architectures influence ionics and electrodics conditions locally, to the impact, in random configurations, of tortuous pathways and outliers in the distributions of local geometries.

The practical necessity to pack nanostructured elements densely together for high energy and power density expands the significance of the additional geometric parameters that the architecture imposes. For example, while the performance of an isolated coaxial nanowire electrode immersed in a sea of electrolyte depends on the material parameters and relative thickness of current collecting and ion storage layers to suitably balance ion and electron transport contributions, densely spaced arrays of such nanoelectrodes specify additional geometric parameters - inter-electrode spacings, number of nearestneighbors, and aspect ratios of electrolyte environments between the nanoelectrodes, even if these are organized as "regular" architectures with fixed spacing and perhaps ordered orientation.

In "random" (or "aperiodic") architectures, dimensional characterization becomes even more complex, introducing parameters such as porosity (volume fraction occupied by electrolyte) and tortuosity (average path length compared to direct line). These parameters play an important role because electrochemical behavior depends at least on both porosity and tortuosity. Furthermore, if important phenomena behave nonlinearly with local inter-electrode spacings, the impact of local variations in architecture could be profound.

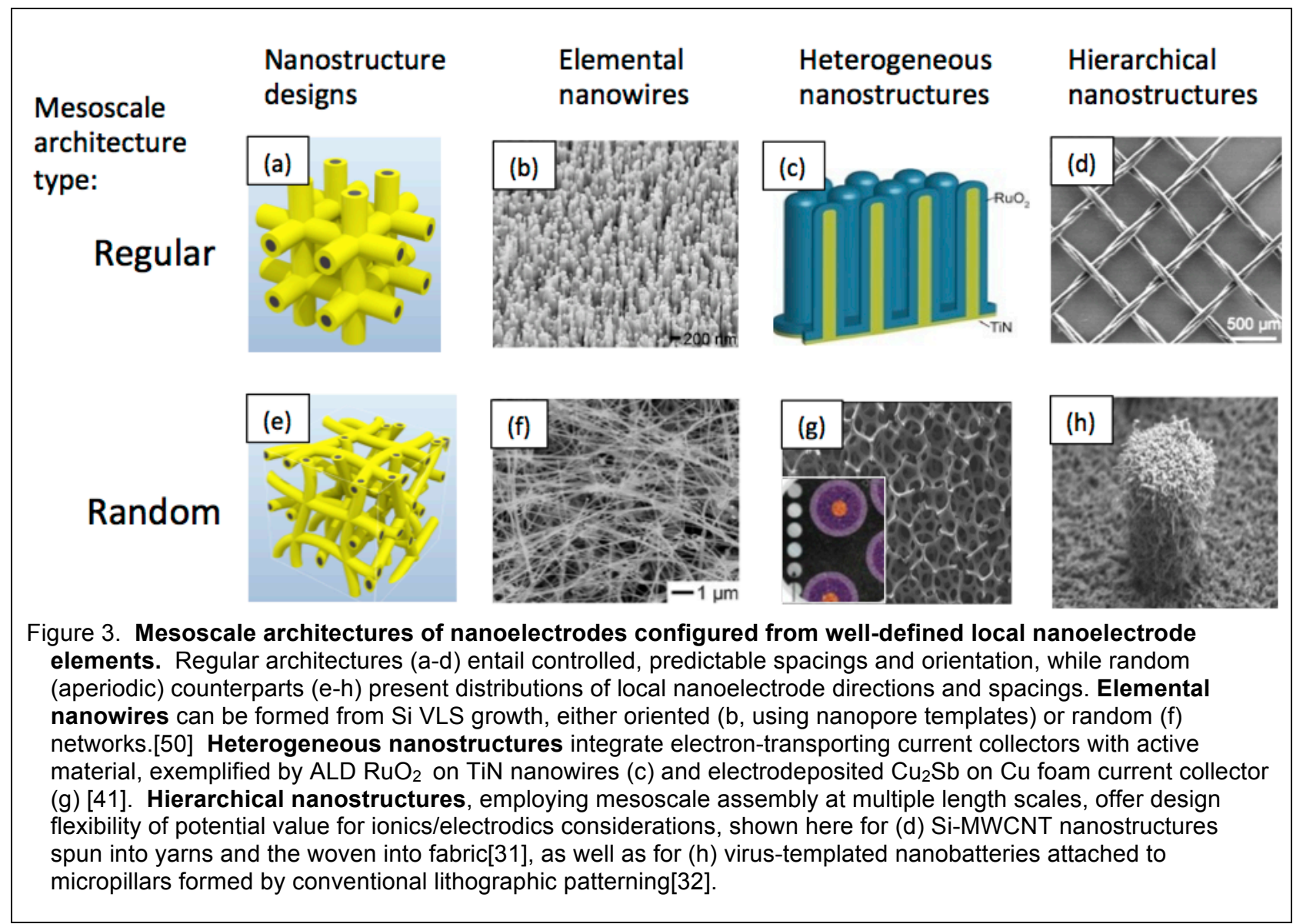

Well-defined nanostructural elements can be assembled into a very diverse set of mesoscale architectures, as depicted for both "regular" and "random" configurations in Fig. 3. By "regular" we refer to extended nanoelectrode networks that are substantially equispaced and parallel (Fig. 3a), whereas by "random" we suggest disordered or amorphous networks (Fig. 3e). Porosity (the volume fraction for electrolyte, not occupied by the nanoelectrode network) is an important parameter for both regular and random architectures. Tortuosity is particularly important for random architectures, describing increases in path length through the structure for ions in the electrolyte and/or electrons in the nanoelectrode[51,52]. 
However, consideration of tortuous networks in electrochemical energy storage highlights a scientific challenge at the mesoscale: geometric path length is unlikely to reflect transport-related performance, because transport "bottlenecks", either flow- or reaction-related, may well determine rates of transport through the porous material. As an example, ions may experience physically small apertures or charge barriers, depending on local electrode geometry encountered or on charge distributions or chemical reaction rates at specific localized positions in the pathway.

Nanostructure synthesis capabilities enable elemental or heterogeneous electrode scaffolds as depicted in figs. 3b, 3c, 3f, and 3g. Hierarchical architectures (Figs. 3d and 3h) amplify the design freedom available at the mesoscale, combining multiple length scales with possibility for more efficient charge transport over extended distances. [37,53]

Such diverse architectures can exhibit quite different performance metrics - gravimetric and volumetric energy density, areal densities, capacity vs. power, etc., as well as capacity maintenance during charge discharge cycling (a degradation issue described below). Each architecture offers a different balance between ion storage, ion transport, and electron transport. Unraveling the science of these relationships in various mesoscale architectures, even assuming the simplest electrochemical and related behavior, is a new mesoscale challenge for modeling and simulation. Mesoscale architectures of nanostructures require multiscale approaches that fuse molecular-scale behavior with mesoscale manifestations of transport through complex architectures ranging from high-aspect ratio regular channels to tortuous geometries characterized by broad parameter distributions. Powerful methods are available in the former case, including various version of ab-initio, quantum chemical and DFT approaches, while continuum methods such as finite element are appropriate for integration at the mesoscale. However, we suggest that there is much more critical innovation and effort to be stimulated and pursued through modeling and simulation for mesoscale architectures and their attendant scientific problems, exemplified by the ionics and electrodics issues described above. For example, geometric aspects of different architectures may produce spatial regions of flow constriction or high current density that stimulate nonlinear chemistry/electrochemistry and local heating which lead to degradation, as described below. Such considerations would stretch well beyond traditional characterization by porosity and tortuosity. Since it is mesoscale architectures that will connect nanoscale advances to engineering advances in energy storage, theoretical and computational efforts along these lines are imperative.

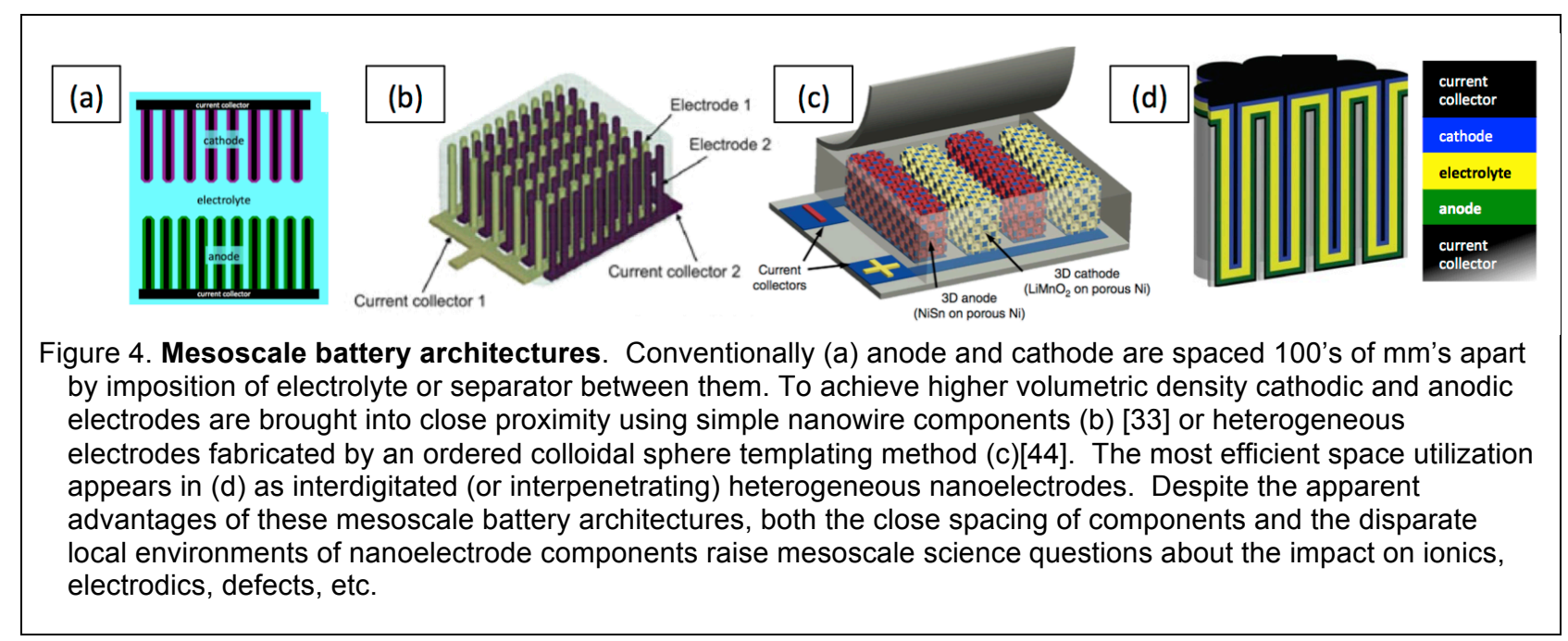

These architectural features persist in the mesoscale architecture of full batteries (i.e., two electrodes), as reflected in Fig. 4. While conventional configurations (Fig. 4a) would involve a micro/macro scale separation between planar or nanostructured electrodes, occupied by electrolyte and separator (not shown), nanostructures may be assembled into more space-efficient configurations with closer electrode-electrode proximity at the micro or nano scale. In Fig. $4 \mathrm{~b}$ anodic and cathodic nanoelectrodes are interspersed in alternating rows with nanoscale spacings between them. The structure in Fig. 4c similarly exploits alternating anode and cathode structures, except that the electrodes at the microscale of nanostructured current collecting, storage, and electrolyte layers formed from a 
sacrificial colloidal template. The interdigitated (interpenetrating) architecture in Fig. 4d represents perhaps the most space-efficient mesoscale architecture, maximizing the volume occupied by active functional components. Here the bottom electrode may be an electrical current collector itself (most space-efficient) or one disposed over an inert scaffold (e.g., nanoporous anodic aluminum oxide).

These design directions - higher surface areas, higher aspect ratios, and likely sharper features (corners, etc.) - certainly represent inhomogeneities and invite other behavioral modes, such as local "hot spots" where ion and/or electron current are enhanced. The impact of such inhomogeneities is an important challenge, and an opportunity for modeling and simulation to provide critical guidance. Early investigations of the impact of nano/micro designs like these are starting to emerge. [27,54]

\section{Defects, outliers, and their evolution}

Defects and outliers are expected and inevitable consequences in dense mesoscale architectures, whether nanoparticulate composites or as precision nanowires, due to the sheer numbers $\left(\sim 10^{10}\right.$ $10^{12} / \mathrm{cm}^{2}$ ) of nanostructures required for any viable energy storage device. Here we use the term "defect" to imply local configurations that are inconsistent with the intended architectural design. By "outliers" we mean configurations that result from the statistical distributions which synthesis and fabrication always generate (e.g., a dimension that is $2 \sigma$ or more different from the mean). Because defects and outliers can impede performance and/or durability, or evolve during operation to do so, they are primary suspects in degradation and failure. Since nanostructures for energy storage will provide value only at the mesoscale integration levels, understanding and controlling defects, outliers, and their evolution into sources of degradation and failure is therefore a critical challenge in the EES domain of mesoscale science.

With nanostructured EES designed to control shapes, dimensions, and material properties of the structures and their spatial relation to each other, it is easy to envision defects and outliers in regular architectures, and even more so for random architectures where a much more diverse set of local network geometries is expected. Examples of defect and outlier sources and their evolution is given in Fig. 5. The colored circles in the Fig. 5 images depict as examples mean (green), above mean (red), and below mean (yellow) values for resulting parameters or structural configurations. A general picture of the statistical distribution of parameters is indicated in the bottom chart of Fig. 5, which features five steps leading to degradation and failure. Here we consider three sources of defects and outliers synthesis, design, and operation - and then the consequences of their evolution in time and during operation.

1. Synthesis of materials, structures, and architectures can incorporate both extrinsic and intrinsic defects. Extrinsic sources include contaminants or particulates (inviting clean room conditions as in microelectronics). More interesting are intrinsic sources. Statistical process variations (image from [55]) reflect the stochastic nature of materials processing steps, while process nonuniformities (adapted from [35]) arise in systematic fashion indicative of the limitations of the process (e.g., for gradients in thickness for layers deposited in nanopores). Once formed, the materials and structures may relax $[38,56]$ into new configurations due to driving forces (e.g., stress) built in through heterogeneous materials, their interfaces, and their shapes.

2. Design of structures and architectures can invite defects and outliers. With dense nanostructure packing, mesoscale packing dimensions may be narrowed too far, leading to nanouniformities and resulting outliers (e.g., sites of excessive current density) and failure. The 3-D geometry of the nanostructures (image from [27]) themselves may be flawed, unable to sustain operating conditions. And with the complexity of 3-D nanogeometries, sharp corners in the design are particularly suspect (image from [57]).

3. Operation of the EES system can certainly generate defects and outliers. One variant arises from stochastic variation, where the natural randomness of molecular-scale chemical events may produce topological or other changes, possibly stimulating even further heterogeneity. For this the growth of $\mathrm{Li}$ dendrites may be a good example. Another type involves physical and/or chemical mechanisms that alter the nanostructures in some way. For EES charge/discharge cycling is a prime driver of changes in structure and architecture, leading to defects and outliers which result in capacity degradation (image from [58]).

Rubloff, Lee: New Science at the Meso Frontier: Dense Nanostructure Architectures for Electrical Energy Storage 
4. Dynamic evolution of defects, outliers, and nonuniformities is really the critical issue. Defects and outliers (\#1 and \#2) present before operation may or may not compromise electrochemical performance, and new ones may be generated during operation (\#3). In any case, all of these are susceptible to change during electrochemical operation, so that such features will evolve dynamically by chemical, electrochemical or physical mechanisms. The statistical distribution in Fig. 5 is symbolic of system parameters - dimensions, rates, local fields, concentration gradients, etc. - with a mean and outliers. As operation continues, the outliers to such distributions tend to proceed at different rates than the mean, leading to increasing breadth of such a distribution and greater heterogeneity is the mesoscale system.

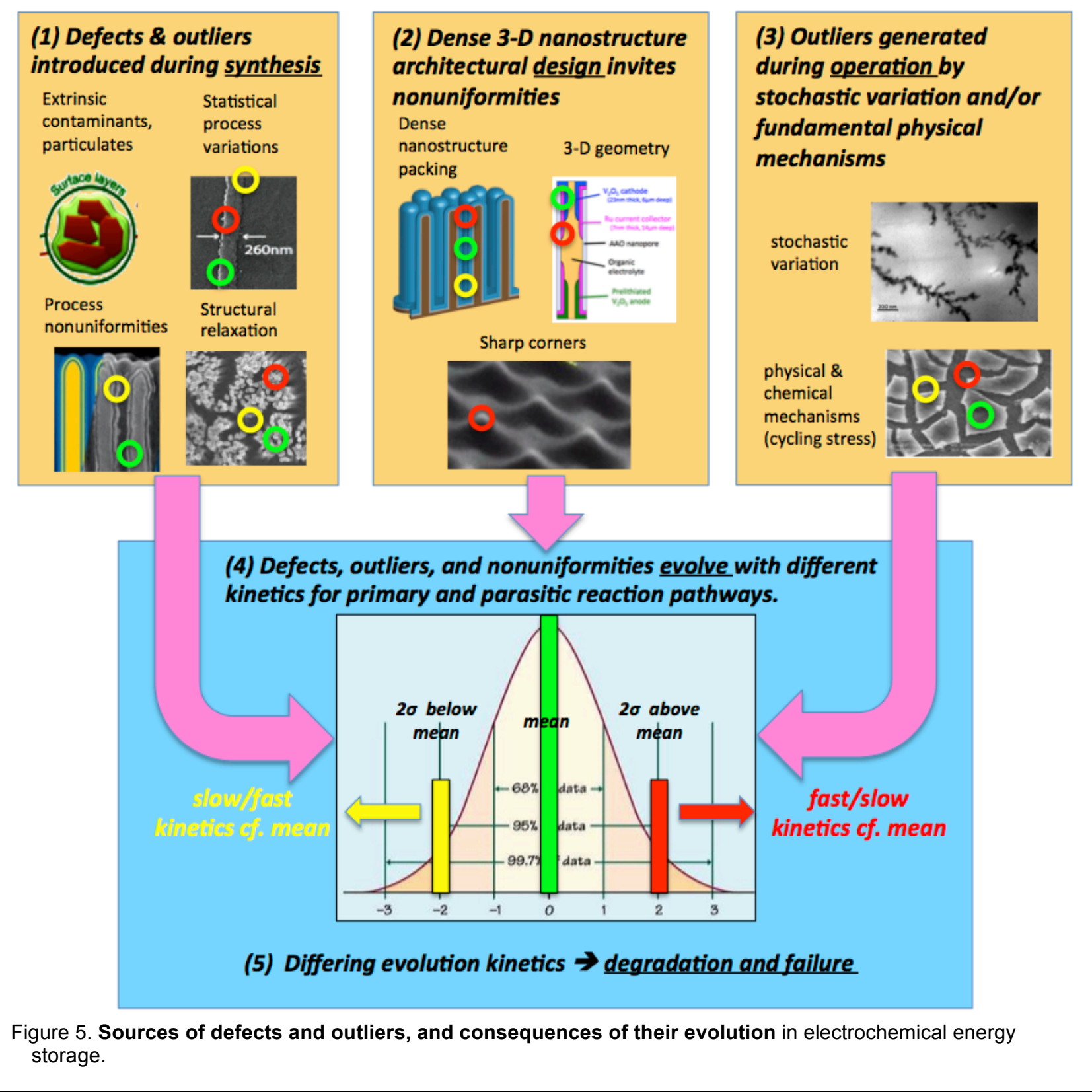

5. Degradation and failure can be consequences of this broadening distribution. For example, when dimensions shrink or fluxes increase sufficiently at a local defect or outlier, capacity degradation or even failure can be expected. One can think of this meaning that such degradation sites become even more dramatically outliers beyond the point in the distribution(s) where degradation and failure occur. Thinking in these terms is analogous to the way yield and reliability are regarded in the field of microelectronic 
devices.

A science of degradation for nanostructures and their mesoscale architectures is required. We envision profound and fundamental science challenges related to degradation and failure in mesoscale EES systems. One is to elucidate the fundamental mechanisms responsible for degradation in EES systems and their relation to molecular, nano, and meso dimensional regimes. The second is to develop modeling and simulation frameworks to treat nanostructure networks assembled into mesoscale architectures, as described in above. The connection between these two may be equally important, where relevant chemistry, electrochemistry, and physics of materials and nanostructures intersect with mesoscale architectural design. Particularly challenging will be nonlinear dependence of the electrochemistry on network dimensions and the tendency for random architectures to produce extreme configurations that serve as degradation initiators. The impact of such a degradation science could be profound for EES capacity maintenance and for safety concerns arising from catastrophic failure.

\section{Concluding thoughts}

Nanostructured electrical energy storage configurations offer profound advantages in terms of power and cycle stability while maintaining high energy density, particularly if emerging ground rules for design of heterogeneous nanostructures are obeyed as massive assemblies of such nanostructures are assembled into regular or random mesoscale architectures. Such massive architectures are prerequisite to a next-generation nanostructure-based storage technology, but they pose a host of deep scientific challenges. Here we highlight three such areas of challenge. First, there may be profound consequences of dense architectures on "ionics" in highly confined nanoenvironments and more generally on ion and electron transport in extended nanoscale networks. Second, we also emphasize the complex tradeoffs between different architectures, where increasingly dense arrangements of active components for higher performance may encounter mechanisms (particularly associated with 3-D spatial complexity) that offset the gains, an issue yet to be adjudicated through research. Finally, we underscore the importance of defects, outliers, and their evolution as instigators of serious degradation, failure, and safety concerns in electrical energy storage devices, which are closely coupled to architectural design and ionics/electrodics.

While anything but easy, experimental efforts to synthesize mesoscale architectures have been rewarding, resulting in a diverse portfolio of configurations exemplified in Figs. 3 and 4 . A central challenge is to design and fabricate unique testbeds that provide insight into operative mechanisms of ionics/electrodics, degradation, and performance of mesoscale architectures, along with creative characterization approaches for these testbeds.

Modeling and simulation face the greatest of opportunities. The connection between nano and macro, bridged by mesoscale science, is largely uncharted territory, though computational tools from molecular to continuum are widely available and continuously improving at a rate far exceeding that of experimental research. The real challenge is to create new ways to achieve viable multi-scale, multi-physics strategies, exploiting reduced-order models and framework linkages that enrich the learning available. To meet this opportunity, it will be important to nurture a new cadre of scientists anxious and capable to fuse knowledge and insight across the mesoscale and to connect closely with experimental advances that can refine lower-order chemistry/physics models while also validating mesoscale models. We note that the modeling and simulation needed at the mesoscale, as assessed here for electrical energy storage, describe many of the attributes of systems engineering - hierarchical models in a framework, reduced order models to describe imperfectly understood chemistry/physics within the larger systems framework, embedded control in the form of chemical/physical rules which govern how synthesis leads to networks with specific characteristics (e.g., connectivity), and optimization methods for complex systems.

Finally, we believe and hope that the central ideas described here for mesoscale energy storage have counterparts in other areas of nanostructure-based energy, including photovoltaics, photoelectrochemistry, and thermoelectrics. These areas all entertain mesoscale architectures of smaller, well-defined nanostructures. Again, in massive assemblies, defects and outliers will play a significant role in degradation and failure. And corresponding to ionics/electrodics here, there will certainly be fundamental consequences of dense packing of nanostructures.

Rubloff, Lee: New Science at the Meso Frontier: Dense Nanostructure Architectures for Electrical Energy Storage 


\section{Acknowledgement}

This work was supported by Nanostructures for Electrical Energy Storage (NEES), an Energy Frontier Research Center funded by the U.S. Department of Energy, Office of Science, Office of Basic Energy Sciences under Award Number DESC0001160.

The authors are profoundly grateful for broad intellectual resonances and welcome discussions with colleagues in the NEES EFRC. It is this community's appetite for scientific growth in new directions that has encouraged the thinking expressed here and made NEES research a pleasure. In addition, we both appreciate having had contact with industry and technology, where the meso issues described here are intrinsically pertinent. Finally, we are grateful to the Basic Energy Sciences Advisory Committee of the Office of Science, which convened the MESO report activity and helped to advance our perspective on the systems aspects of mesoscale science.

\section{References}

[1] BESAC. From Quanta to the Continuum: Opportunities for Mesoscale Science 2012:1-86.

[2] Thackeray MM, Wolverton C, Isaacs ED. Electrical energy storage for transportationapproaching the limits of, and going beyond, lithium-ion batteries. Energ Environ Sci 2012;5:7854.

[3] Thackeray MM, Kang SH, Johnson CS, Vaughey JT, Hackney SA. Comments on the structural complexity of lithium-rich $\mathrm{Li} 1+\mathrm{xM} 1-\mathrm{xO} 2$ electrodes $(\mathrm{M}=\mathrm{Mn}, \mathrm{Ni}, \mathrm{Co})$ for lithium batteries. Electrochem Commun 2006;8:1531-8.

[4] Armand M, Tarascon JM. Building better batteries. Nature 2008;451:652-7.

[5] Amatucci G, Tarascon JM. Optimization of insertion compounds such as LiMn2O4 for Li-ion batteries. J Electrochem Soc 2002;149:K31-K46.

[6] Arico AS, Bruce P, Scrosati B, Tarascon JM, Van Schalkwijk W. Nanostructured materials for advanced energy conversion and storage devices. Nat Mater 2005;4:366-77.

[7] Sides CR, Li NC, Patrissi CJ, Scrosati B, Martin CR. Nanoscale materials for lithium-ion batteries. MRS Bulletin 2002;27:604-7.

[8] Marom R, Amalraj SF, Leifer N, Jacob D, Aurbach D. A review of advanced and practical lithium battery materials. J Mater Chem 2011;21:9938.

[9] Yoo HD, Shterenberg I, Gofer Y, Gershinsky G, Pour N, Aurbach D. Mg rechargeable batteries: an on-going challenge. Energy Environ Sci 2013;6:2265-79.

[10] Zhou H, Nanda J, Martha SK, Adcock J, Idrobo JC, Baggetto L, et al. Formation of Iron Oxyfluoride Phase on the Surface of Nano-Fe 30 4Conversion Compound for Electrochemical Energy Storage. J Phys Chem Lett 2013;4:3798-805.

[11] Ban CM, Li Z, Wu ZC, Kirkham MJ, Chen L, Jung YS, et al. Extremely Durable High-Rate Capability of a $\mathrm{LiNi}(0.4) \mathrm{Mn}(0.4) \mathrm{Co}(0.2) \mathrm{O}(2)$ Cathode Enabled with Single-Walled Carbon Nanotubes. Advanced Energy Materials 2011;1:58-62.

[12] Omenya F, Chernova NA, Zhang R, Fang J, Huang Y, Cohen F, et al. Why Substitution Enhances the Reactivity of LiFePO4. Chem Mater 2013;\{25\}:\{85-89\}.

[13] Takeuchi ES, Marschilok AC, Takeuchi KJ. Secondary Battery Science: At the Confluence of Electrochemistry and Materials Engineering. Electrochemistry 2012;\{80\}:\{700-705\}.

[14] Liao H, Karki K, Zhang Y, Cumings J, Wang Y. Interfacial Mechanics of Carbon Nanotube@Amorphous-Si Coaxial Nanostructures. Adv Mater 2011;23:4318-22.

[15] Deng S, Zhang Y, Brozena AH, Mayes ML, Banerjee P, Chiou W-A, et al. Confined propagation of covalent chemical reactions on single-walled carbon nanotubes. Nat Commun 2011;2:382.

[16] Chen X, Gerasopoulos K, Guo J, Brown A, Wang C, Ghodssi R, et al. Virus-Enabled Silicon Anode for Lithium-Ion Batteries. Acs Nano 2010;4:5366-72.

[17] Li NC, Martin CR, Scrosati B. Nanomaterial-based Li-ion battery electrodes. J Power Sources 2001;97-8:240-3.

[18] Sides CR, Martin CR. Nanostructured electrodes and the low-temperature performance of Li-ion batteries. Adv Mater 2005;17:125-.

[19] Ruzmetov D, Oleshko VP, Haney PM, Lezec HJ, Karki K, Baloch KH, et al. Electrolyte Stability Determines Scaling Limits for Solid-State 3D Li Ion Batteries. Nano Lett 2011;12:505-11.

[20] Karki K, Zhu Y, Liu Y, Sun CF, Hu L, Wang Y, et al. Hoop-Strong Nanotubes for Battery 
[21] Sun CF, Karki K, Jia Z, Liao H, Zhang Y, Li T, et al. A beaded-string silicon anode. Acs Nano 2013;7:2717-24.

[22] Sherrill SA, Duay J, Gui Z, Banerjee P, Rubloff GW, Lee SB. MnO2/TiN heterogeneous nanostructure design for electrochemical energy storage. Physical Chemistry Chemical Physics : PCCP 2011;13:15221-6.

[23] Sherrill SA, Banerjee P, Rubloff GW, Lee SB. High to ultra-high power electrical energy storage. Physical Chemistry Chemical Physics : PCCP 2011;13:20714-23.

[24] Chen X, Zhu H, Chen Y-C, Shang Y, Cao A, Hu L, et al. MWCNT/V 20 5Core/Shell Sponge for High Areal Capacity and Power Density Li-lon Cathodes. Acs Nano 2012;6:7948-55.

[25] Rubloff GW, Kozen AC, Bok Lee S. From nanoscience to solutions in electrochemical energy storage. J Vac Sci Technol A 2013;31:058503.

[26] Martha SK, Nanda J, Zhou H, Idrobo JC, Dudney NJ, Pannala S, et al. Electrode architectures for high capacity multivalent conversion compounds: iron (ii and iii) fluoride. RSC Adv 2014;4:6730.

[27] Liu C, Gillette E, Chen X, Pearse A, Kozen AC, Schroeder MA, et al. All-in-one Nanopore Battery Array. (In Review for Nature Nanotechnology) n.d.

[28] Long JW, Rolison DR. Architectural design, interior decoration, and three-dimensional plumbing en route to multifunctional nanoarchitectures. Accounts Chem Res 2007;40:854-62.

[29] Rolison DR, Long RW, Lytle JC, Fischer AE, Rhodes CP, McEvoy TM, et al. Multifunctional 3D nanoarchitectures for energy storage and conversion. Chem Soc Rev 2009;38:226-52.

[30] Rolison DR, Nazar LF. Electrochemical energy storage to power the 21st century. MRS Bulletin 2011;36:486-93.

[31] Sun C-F, Zhu H, Baker lii EB, Okada M, Wan J, Ghemes A, et al. Weavable high-capacity electrodes. Nano Energy 2013;2:987-94.

[32] Gerasopoulos K, Pomerantseva E, McCarthy M, Brown A, Wang C, Culver J, et al. Hierarchical Three-Dimensional Microbattery Electrodes Combining Bottom-Up Self-Assembly and TopDown Micromachining. Acs Nano 2012;6:6422-32.

[33] Arthur TS, Bates DJ, Cirigliano N, Johnson DC, Malati P, Mosby JM, et al. Three-dimensional electrodes and battery architectures. MRS Bulletin 2011;36:523-31.

[34] Long JW, Dunn B, Rolison DR, White HS. Three-dimensional battery architectures. Chem Rev 2004;104:4463-92.

[35] Banerjee P, Perez I, Henn-Lecordier L, Lee SB, Rubloff GW. Nanotubular metal-insulator-metal capacitor arrays for energy storage. Nat Nanotechnol 2009;4:292-6.

[36] Chen X, Zhu H, Liu C, Chen Y-C, Weadock N, Rubloff G, et al. Role of mesoporosity in cellulose fibers for paper-based fast electrochemical energy storage. J Mater Chem A 2013;1:8201.

[37] Gui Z, Zhu HL, Gillette E, Han XG, Rubloff GW, Hu LB, et al. Natural Cellulose Fiber as Substrate for Supercapacitor. Acs Nano 2013;7:6037-46.

[38] Liu R, Duay J, Lee SB. Heterogeneous nanostructured electrode materials for electrochemical energy storage. Chemical Communications 2011;47:1384-404.

[39] Gregorczyk K, Banerjee P, Rubloff GW. Conduction in ultrathin ruthenium electrodes prepared by atomic layer deposition. Materials Letters 2012;73:43-6.

[40] IUPAC. Compendium of Chemical Terminology, 2nd ed. (the "Gold Book"). Blackwell Scientific Publications, Oxford (1997); n.d.

[41] Johnson DC, Prieto AL. Three-dimensional lithium-ion batteries with interdigitated electrodes. Proceedings of SPIE 2013:1-10.

[42] Chae W-S, Gough DV, Ham S-K, Robinson DB, Braun PV. Effect of Ordered Intermediate Porosity on Ion Transport in Hierarchically Nanoporous Electrodes. ACS Appl Mater Interfaces 2012;4:3973-9.

[43] Zhang H, Yu X, Braun PV. Three-dimensional bicontinuous ultrafast-charge and -discharge bulk battery electrodes. Nat Nanotechnol 2011;6:277-81.

[44] Pikul JH, Zhang HG, Cho J, Braun PV, King WP. High-power lithium ion microbatteries from interdigitated three-dimensional bicontinuous nanoporous electrodes. Nat Commun $1 \mathrm{AD} ; 4: 1732-5$.

[45] White RJ, White HS. Electrochemistry in Nanometer-Wide Electrochemical Cells. Langmuir 2008;24:2850-5.

Rubloff, Lee: New Science at the Meso Frontier: Dense Nanostructure Architectures for Electrical Energy Storage 
[46] Siwy Z, Kosinska ID, Fulinski A, Martin CR. Asymmetric diffusion through synthetic nanopores. Phys Rev Lett 2005;94.

[47] Jin P, Mukaibo H, Horne LP, Bishop GW, Martin CR. Electroosmotic Flow Rectification in Pyramidal-Pore Mica Membranes. Journal of the American Chemical Society 2010;132:2118-9.

[48] Nishizawa M, Menon VP, Martin CR. Metal Nanotubule Membranes with Electrochemically Switchable Ion-Transport Selectivity. Science 1995;268:700-2.

[49] Pevarnik M, Healy K, Davenport M, Yen J, Siwy ZS. A hydrophobic entrance enhances ion current rectification and induces dewetting in asymmetric nanopores. The Analyst 2012;137:2944-50.

[50] Cho J-H, Picraux ST. Enhanced Lithium Ion Battery Cycling of Silicon Nanowire Anodes by Template Growth to Eliminate Silicon Underlayer Islands. Nano Lett 2013;13:5740-7.

[51] Ebner M, Chung D-W, Garcia RE, Wood V. Tortuosity Anisotropy in Lithium-lon Battery Electrodes. Advanced Energy Materials 2014;\{4\}.

[52] Vijayaraghavan B, Ely DR, Chiang Y-M, Garcia-Garcia R, Garcia RE. An Analytical Method to Determine Tortuosity in Rechargeable Battery Electrodes. J Electrochem Soc 2012; $\{159\}:\{$ A548-A552\}.

[53] Chen X, Zhu H, Liu C, Chen Y-C, Weadock N, Rubloff G, et al. Role of mesoporosity in cellulose fibers for paper-based fast electrochemical energy storage. J Mater Chem A 2013;1:8201.

[54] Zadin V, Kasemägi H, Aabloo A, Brandell D. Modelling electrode material utilization in the trench model 3D-microbattery by finite element analysis. J Power Sources 2010;195:6218-24.

[55] Yan W, Ayvazian T, Kim J, Liu Y, Donavan KC, Xing W, et al. Mesoporous Manganese Oxide Nanowires for High-Capacity, High-Rate, Hybrid Electrical Energy Storage. Acs Nano 2011;5:8275-87.

[56] Liu R, Duay J, Lee SB. Redox Exchange Induced MnO2 Nanoparticle Enrichment in Poly(3,4ethylenedioxythiophene) Nanowires for Electrochemical Energy Storage. Acs Nano 2010;4:4299-307.

[57] Haspert LC, Lee SB, Rubloff GW. Nanoengineering strategies for metal-insulator-metal electrostatic nanocapacitors. Acs Nano 2012;6:3528-36.

[58] Kasavajjula U, Wang C, Appleby AJ. Nano-and bulk-silicon-based insertion anodes for lithiumion secondary cells. J Power Sources 2007;163:1003-39.

Rubloff, Lee: New Science at the Meso Frontier: Dense 